\title{
HIGH-GAIN HARMONIC GENERATION FREE-ELECTRON LASER AT SATURATION*
}

\author{
T. Shaftan ${ }^{1}$, M. Babzien, I. Ben-Zvi, S. G. Biedron ${ }^{\dagger}$, L. F. DiMauro, A. Doyuran, W. Graves, \\ J. Jagger ${ }^{\dagger}$, E. Johnson, S. Krinsky, R. Malone, I. Pogorelsky, V. Sajaev ${ }^{\dagger}$, B. Sheehy, \\ J. Skaritka, L. Solomon, G. Rakowsky, I. Vasserman ${ }^{\dagger}$, J.H. Wu, X.J. Wang, \\ M. Woodle, V. Yakimenko, L.-H. Yu \\ Brookhaven National Laboratory, Upton, New York 11973 \\ ${ }^{\dagger}$ Advanced Photon Source, Argonne National Laboratory, Argonne, Illinois 60439
}

\section{Abstract}

We report on observations of the output of a high-gain harmonic-generation (HGHG) free-electron laser (FEL) at saturation. A seed $\mathrm{CO}_{2}$ laser at a wavelength of $10.6 \mu \mathrm{m}$ was used to generate amplified FEL output at $5.3 \mu \mathrm{m}$. Measurement of the frequency spectrum, pulse duration and correlation length of the $5.3-\mu \mathrm{m}$ output verified the longitudinal coherence of the light. At the exit of the device, measurements of the energy distribution of the electron beam indicated that the HGHG process had reached saturation. By comparing the intensities of the higher harmonics $(2.65 \mu \mathrm{m}$ and $1.77 \mu \mathrm{m})$ relative to the $5.3-\mu \mathrm{m}$ fundamental, additional evidence confirming saturated operation was obtained. These results agree with theoretical predictions at saturation.

We also briefly describe the status of a new HGHG experiment at the Source Development Laboratory (BNL), aimed at achieving generation of 100-nm radiation.

\section{INTRODUCTION}

In the HGHG scheme [1] a coherent seed at a wavelength that is a subharmonic of the desired output radiation interacts with the electron beam in an energymodulating section. This energy modulation is then converted into spatial bunching while traversing a dispersive section. In the second undulator (the radiator), which is tuned to a higher harmonic of the seed radiation, the microbunched electron beam first emits coherent radiation and then amplifies it exponentially until saturation is achieved.

A schematic of the HGHG Accelerator Test Facility (ATF) layout [2] with electron beam, seed laser, and calculated $\mathrm{HGHG}$ radiation parameters are shown in Fig. 1.

The frequency-quadrupled Nd:YAG photocathode-rf gun drive laser has a pulse length of 8 ps and an energy of $8 \mu \mathrm{J}$, resulting in a 6-ps FWHM electron beam pulselength with a maximum of $130 \mathrm{~A}$ peak current off the $\mathrm{Mg}$ cathode. The electron beam is accelerated via two $3-\mathrm{m}$, SLAC-type, constant-gradient accelerating structures before being bent to enter the ATF experimental all where it is introduced to the HGHG beamline after passage through a second dipole magnet. This bending

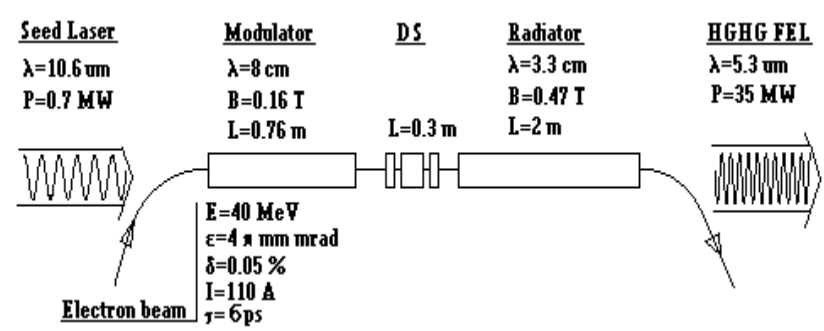

Fig. 1: HGHG ATF layout.

dipole also allows an area where the $\mathrm{CO}_{2}$ seed laser can be introduced. The seed laser delivers $4 \mathrm{~mJ}$ at a $10.6-\mu \mathrm{m}$ wavelength every 20 seconds.

\section{THE MEASUREMENTS OF HGHG RADIATION PROPERTIES}

We have performed a sequence of the experiments to measure the main parameters of a single HGHG radiation pulse, such as frequency spectrum and coherence length, pulse length, and higher harmonic content.

We used an optical spectrometer and thermal camera to measure a single-shot HGHG spectrum. The multishot and single-shot spectra are shown in Figs. 2(A) and 2(B), respectively.

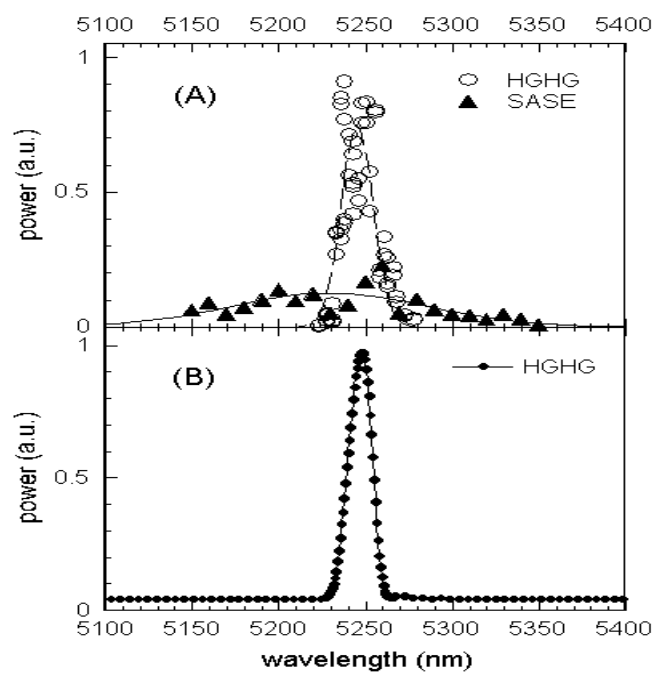

Fig. 2: The multishot and single-shot spectra. 
Figure 2(A) shows the multishot spectra based on the SASE signal $\times 10^{6}$, where every point was averaged over ten shots, as well as the HGHG signal where no averaging was used when obtained using a high-gain, In-Sb detector. The bottom figure shows the single-shot HGHG spectrum obtained using the thermal imager described above. The resolution of this experiment is $2 \mathrm{~nm}$.

For investigation of the time coherence of the HGHG radiation we have used a Michelson interferometer. The mirror in one arm was tilted off-axis, and by changing the angle of tilt we controlled the number of periods in the interference pattern. The contrast of interference was measured by the thermal imaging camera. In order to collect more light, we used a cylindrical mirror to produce a line-type image on the thermal viewer. The camera's images are shown in Fig. 3.
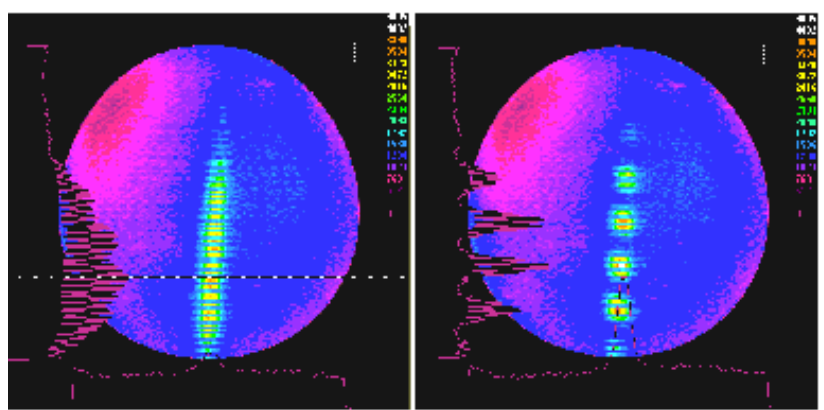

Fig. 3: Interference fringes images.

The images correspond to the wing of the HGHG pulse (left image, visibility of the fringes is low) and center of the pulse (right image, visibility is high).

In order to simplify the alignment and tuning of the interferometer, we used an auxiliary HeNe laser $(0.63 \mu \mathrm{m}$ wavelength) to produce an interference pattern in visible light.

The dependence of visibility $\left(\left(\mathrm{I}_{\max }-\mathrm{I}_{\min }\right) /\left(\mathrm{I}_{\max }+\mathrm{I}_{\min }\right)\right)$ versus delay between two arms for the HGHG pulse measurement is shown in Fig. 4.

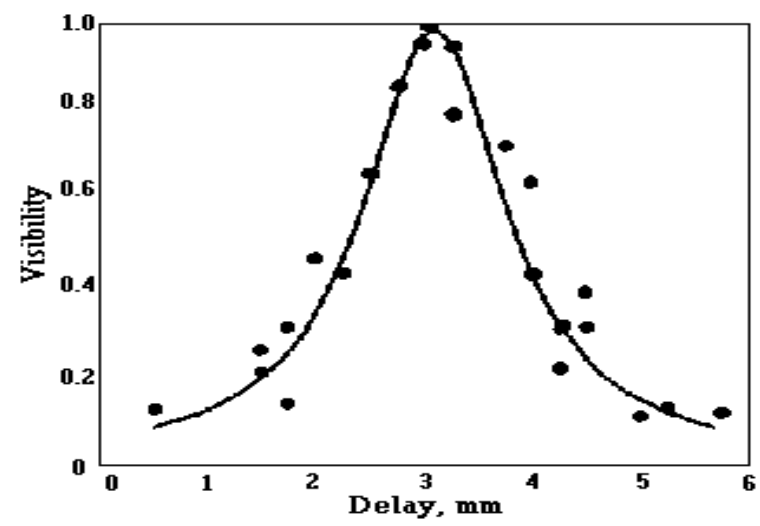

Fig. 4: The dependence of visibility versus delay between two arms.

The optical coherence length was measured as $1.6 \mathrm{~mm}$, or $5.3 \mathrm{ps}$, based upon the delay change.
We used an intensity autocorrelator employing a second harmonic generation in a $\mathrm{AgGaSe}_{2}$ nonlinear crystal. The intensity of the second harmonic was measured by a single-element InSb photoconductive detector. The main sources of error in the measurement are imposed by the low-duty cycle ( 1 shot per 20 seconds) of the $\mathrm{CO}_{2}$ laser and instabilities in the electron beam. In order to reduce scatter, each data point is a single-shot measurement of the second harmonic signal normalized to the square of the fundamental energy. The normalized signal versus delay time (relative length difference between the two arms) is shown in Fig. 5. Assuming a Gaussian pulse shape, the duration is found to be $8.4 / \sqrt{ } 2=5.9 \pm 0.7 \mathrm{ps}$.

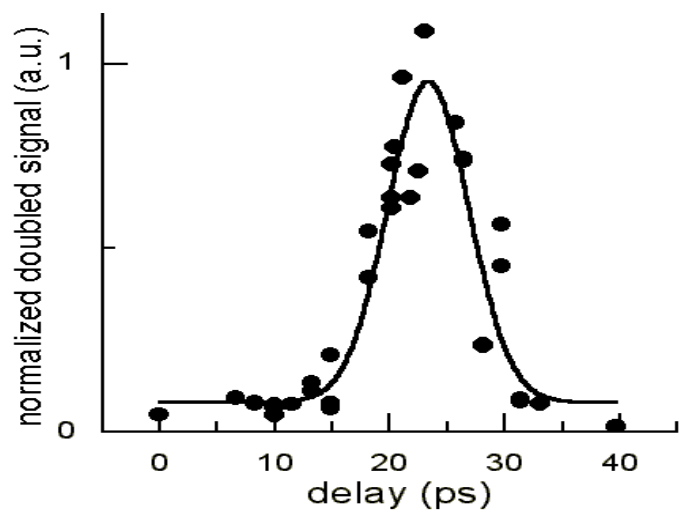

Fig. 5: The second harmonic signal versus optical delay.

The electron beam energy modulation was determined using the electron energy spectrometer after the radiator section. Energy modulation of the electron beam is generated in two ways in the HGHG process: through the initial interaction of the seed laser in the modulator and through the HGHG FEL interaction itself in the radiator. The energy modulation in the radiator predominates and the electron beam image after the spectrometer (a) without and (b) with the $\mathrm{CO}_{2}$ beam present, is shown in Fig. 6 . The latter shows an energy modulation of nearly $2.5 \%$ FWHM.

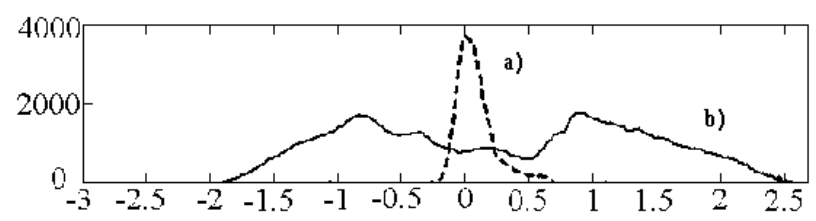

Fig. 6: The energy modulation (electron beam distribution after spectrometer, horizontal axis in \% of energy spread).

The amount of modulation, as well as the existence of the higher harmonics, reveals that saturation or nearsaturation has been reached. The higher harmonics have been previously predicted in theory [3]. We measured the fundamental $(5.3 \mu \mathrm{m})$, second $(2.65 \mu \mathrm{m})$, and third $(1.77$ $\mu \mathrm{m})$ harmonics as a function of modulation described above using the InSb detector in conjunction with the appropriate bandpass and neutral density filters to produce similar signal levels on the detector. 
In Fig. 7, the harmonic power $(\mu \mathrm{J})$ versus electron beam energy modulation (\%) is shown. In Table 1, the theoretical and experimentally measured harmonics to fundamental ratios are shown. There is a good agreement between experiment and theory.

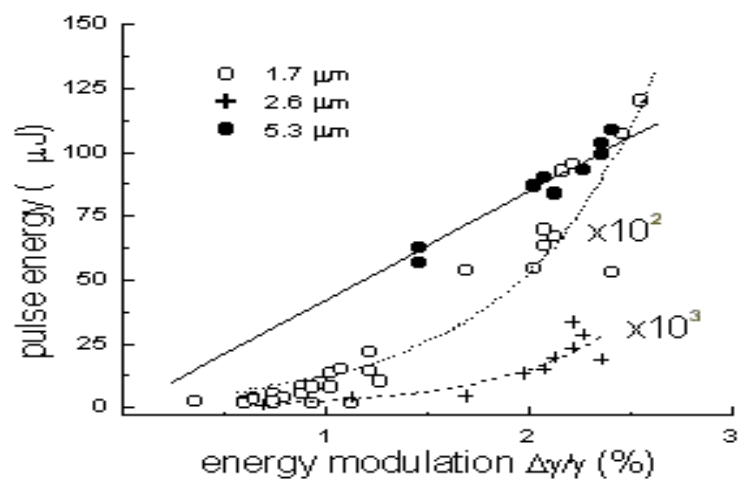

Fig. 7: The harmonic power versus FWHM modulation.

Table 1. Harmonic content of the HGHG radiation.

\begin{tabular}{|l|l|l|}
\hline Wavelength & Simulation & Experiment \\
\hline $2.65 \mu \mathrm{m}$ & $6 \times 10^{-4}$ & $2 \times 10^{-4}$ \\
\hline $1.77 \mu \mathrm{m}$ & $1 \times 10^{-2}$ & $8 \times 10^{-3}$ \\
\hline
\end{tabular}

\section{THE DUVFEL PROJECT}

The Deep Ultra Violet Free Electron Laser (DUVFEL) in the Source Development Laboratory (SDL) [4] is the next step in the development of a short-wavelength HGHG FEL. The DUVFEL research plan includes three stages to generate a coherent radiation of $400-\mathrm{nm}, 200$ $\mathrm{nm}$, and, after energy upgrade, $100-\mathrm{nm}$ coherent radiation.

The layout of the SDL facility is shown in Fig. 8. The accelerator consists of a BNL/SLAC/UCLA-type electron gun driven by a Ti:Sa laser, four SLAC-type linac structures, and a four-magnet chicane. A $4.3-\mathrm{MeV}$ electron beam leaves the gun, is accelerated up to $60 \mathrm{MeV}$ in two linac sections, and compressed in a magnetic chicane. Final acceleration up to $140 \mathrm{MeV}$ is achieved in the last two linac sections.

The SDL accelerator was commissioned last year. The photocathode-rf gun currently can produce 2-ps (rms) bunches with $300 \mathrm{pC}$ of charge. $4-\pi$-mm-mrad emittances are measured after acceleration up to $75 \mathrm{MeV}$ in the first two linac sections. After compression we obtained a 0.3ps-long bunch with the same transverse emittances and a 300-A peak current.
The FEL magnetic system consists of a mini-undulator, dispersive section, and 10-m-long NISUS undulator [5]. During the first stage of experiments $(400 \mathrm{~nm})$ the harmonic of the Ti:Sa laser will be used as a seed for the HGHG experiment. In order to introduce seed radiation to the vacuum chamber, we discussed two schemes: a "small" four-magnet chicane, that "bends" the electron beam trajectory around the laser mirror, and an axicon mirror [6]. The second solution is more attractive because of the reduced space requirements and the simplification of electron beam optics.

For electron beam and radiation diagnostics in the NISUS we are going to use 16 pop-in monitors with YAG screens and OTR mirrors. Five of them will be used for FEL radiation measurements, and the other five will have shadow shields for OTR measurement to block the spontaneous radiation. The optical spectrometer is installed at the end of the wiggler for the spectrum measurements.

\section{ACKNOWLEDGEMENTS}

We thank all ATF and DUVFEL personnel for their great help in making this experiment successful. This work was supported by U.S. Department of Energy, Office of Basic Energy Sciences, under Contracts Nos. DE-AC02-98CH10886 and W-31-109-ENG-38 and by Office of Naval Research Grant no. N00014-97-1-0845.

\section{REFERENCES}

[1] L. H. Yu, Phys. Rev. A, 44, 5178 (1991).

[2] L. H. Yu et al., Science, 289, 932 (2000).

[3] S.G. Biedron, H.P. Freund, X.J. Wang, L.-H. Yu and S.V. Milton, "Nonlinear Harmonics in the High-Gain Harmonic Generation (HGHG) Experiment," Proc. FEL 2000, Durham, North Carolina, August 13-18, 2000.

[4] L. H. Yu et al., "The DUV-FEL Development Program," these proceedings.

[5] D.C. Quimby et al., "Development of a 10-meter Wedged-pole undulator," NIM A 285, 281 (1989).

[6] I.V. Pogorelsky and W.D. Kimura, "Waveguiding by axicon focused laser beams," Adv. Accel. Concepts $1994,419-428$.

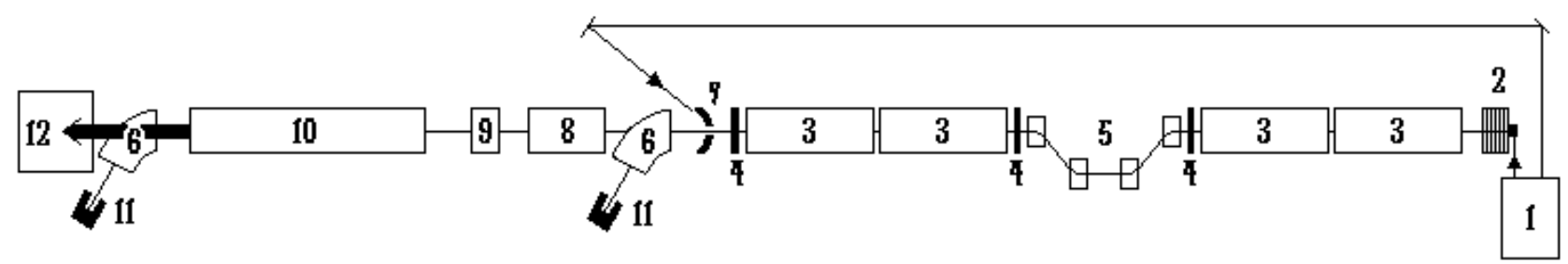

Fig. 8: The layout of SDL. 1 -rf gun and seed laser system, 2 - rf gun, 3 - linac tanks, 4 - focusing triplets, 5 - magnetic chicane, 6 - spectrometers dipoles, 7 - axicon mirror, 8 - mini-undulator, 9 - dispersive section, 10 - NISUS wiggler, 11 - beam dumps, 12 - FEL radiation measurements area. 